\title{
Additional technician tasks and turnaround time in the clinical Stat laboratory
}

\author{
Maria Salinas ${ }^{* 1,2}$, Maite López-Garrigós ${ }^{1,2}$, Emilio Flores ${ }^{1,3}$, Maria Leiva-Salinas², Rosa Lillo, Carlos Leiva-Salinas ${ }^{4}$ \\ ${ }^{1}$ Clinical Laboratory, Hospital Universitario de San Juan, San Juan de Alicante, Spain \\ ${ }^{2}$ Department of Biochemistry and Molecular Pathology, Universidad Miguel Hernandez, Elche, Spain \\ ${ }^{3}$ Department of Clinical Medicine, Universidad Miguel Hernandez, Elche, Spain \\ ${ }^{4}$ Department of Radiology, University of Virginia, Charlottesville, VA, USA \\ *Corresponding author: salinas_mar@gva.es
}

\begin{abstract}
Introduction: Many additional tasks in the Stat laboratory (SL) increase the workload. It is necessary to control them because they can affect the service provided by the laboratory. Our aim is to calculate these tasks, study their evolution over a 10 year period, and compare turnaround times (TAT) in summer period to the rest of the year.

Materials and methods: Additional tasks were classified as "additional test request" and "additional sample". We collected those incidences from the laboratory information system (LIS), and calculated their evolution over time. We also calculated the monthly TAT for troponin for Emergency department (ED) patients, as the difference between the verification and LIS registration time. A median time of 30 minutes was our indicator target. TAT results and tests workload in summer were compared to the rest of the year.

Results: 0ver a 10-year period, the technologists in the SL performed 51,385 additional tasks, a median of 475 per month. The workload was significantly higher during the summer ( 45,496 tests) than the rest of the year $(44,555$ tests) $(P=0.019)$. The troponin TAT did not show this variation between summer and the rest of the year, complying always with our 30 minutes indicator target.

Conclusion: The technicians accomplished a significant number of additional tasks, and the workload kept increasing over the period of 10 years. That did not affect the TAT results.
\end{abstract}

Key words: medical laboratory personnel; quality indicators; health care

\section{Introduction}

The clinical laboratory has always played a critical role in the Emergency department (ED) (1). In recent years, there has been a progressive and significant increase in the request for urgent laboratory tests (ULT) $(2,3)$. At the same time there is more pressure to achieve targets in STAT tests turnaround time (TAT) $(3,4)$. Although decreasing TAT diminishes ED patient length of stay and increases physicians' satisfaction (1), it may result in an increase of the errors rate (5); which is an important consideration, especially when dealing with ED patients that are waiting for a prompt therapeutic decision.
It is well known that TAT is a useful marker of laboratory efficiency, and that process control reduces the laboratory TAT (6). Consequently, assessing the Stat laboratory (SL) technician's workload, to adapt the laboratory resources to increasing demand and TAT requirements (7), is a key element. To do so, one needs to calculate the number of ED patients that undergo laboratory tests and the number of tests requested; but also to identify any additional task the technicians need to perform, regarding additional or late requests from the ED for those same individuals. The latter has not been systematically investigated. 
The hypothesis of the study was that a significant number of additional tasks present in SL may increase and potentially impact turnaround times. The aim of the study was to calculate the number of additional tasks regarding the request of additional tests or submission of additional samples conducted by SL technicians, study its evolution over a 10-year period, and compare TAT in summer period to the rest of the year.

\section{Materials and methods}

\section{Materials}

The SL is an independent laboratory located in the main clinical laboratory at the Hospital de San Juan (396 beds), a general hospital in Alicante, Spain. The laboratory serves a population of 234,403 inhabitants. In year 2015, 86,114 outpatients were treated in the ED. The average of outpatients attended by month during the year was 6909 and 7987 in summer.

$4,962,620$ tests were processed in the SL from January $1^{\text {st }}, 2006$ to March $31^{\text {st }}, 2015.10$ technicians staffed the SL until 2010; it is currently staffed by 11 technicians who process, verify, and report every hematology, coagulation, blood gas, urinalysis and chemistry test for inpatients and ED patients. They are organized into three shifts (3, 2 and 2 technician in the morning, afternoon and night shifts, respectively; except Saturdays and Sundays when there are only 2 technicians in morning shift). An annual session was held between the laboratory staff and ED physicians regarding the education of a better use of SL utilization.

\section{Methods}

In January 2006 a meeting between SL technicians, the pathologist in charge of the SL and the laboratory director was held in order to identify and collect the different additional tasks the SL technologists need to go through daily and that are freely chosen by ED physician. There are not agreements between laboratory and casualty department. The goal was collection of this data as simply and as automatically as possible so that po- tential corrective actions regarding efficiency and TAT could be potentially implemented (8).

The additional tasks were classified as follows: "first additional test request" - when supplemental test(s) were requested from the ED from the same patient sample after the results of a first battery of tests had already been sent (e.g. request of lipase after complete blood count and C-Reactive Protein); and "additional sample" - when a different sample was sent to the laboratory to perform additional tests after the results of tests from the first specimen had already been analysed. (e.g. urine sent after blood sample already analysed). "Second additional test request" and "third additional test request" refer to the same principle as for "first additional test request", when a second or third additional test/series of tests were ordered after additional results have already been sent. In case of occurrence of the previously described situations, the SL technicians were instructed to register the incidence in the Laboratory Information System (LIS).

The period of data collection was from January 1 , 2006 to March 31, 2015. We retrospectively automatically collected the additional workload registers via our LIS, using a data warehouse software program based on On-Line Analytical Processing (OLAP) cubes (Omnium by Roche Diagnostics ${ }^{\circledR}$ SUNSET Technologies, Gerona, Spain). We calculated the absolute number of additional tasks and its rate per 1000 stat laboratory tests for each of the 111 months of the study period. Results for July, August and September, which is traditionally considered vacation time in Spain, possibly due good weather, were compared to the rest of the year. We also calculated the monthly TAT for troponin for ED patients, as the difference between the verification and LIS registration time $(3,4)$. A median time of 30 minutes is our indicator target.

\section{Statistical analysis}

Statistical analyses were performed with the software SPSS version 20 for windows (SPSS Inc, Chicago, IL). The differences in additional tasks, TAT results and workload between summer period and the rest of the year were calculated through 
the U-Mann Whitney test. A two-sided $\mathrm{P} \leq 0.05$ rule was used as the criterion for rejecting the null hypothesis of no difference.

\section{Results}

During the study period, the technologists in the SL performed 51,385 additional tasks, a median of 475 per month. Table 1 shows the descriptive analysis of total and specific additional tasks, expressed monthly in absolute numbers and per 1000 tests requested. Figure 1 shows in a monthly basis, the number of two major additional activities along the period of study.

Table 2 shows that during the summer, the workload was significantly higher than during the rest of the year. The number of additional tasks per 1000 test was also significantly higher in summer.

TABLE 1. Additional tasks performed by the stat laboratory technicians per month.

\begin{tabular}{lc}
\hline Additional tasks per month, median (interquartile range) \\
\hline Total & $475(428-511)$ \\
Total / 1000 tests performed & $10.2(9.6-11.2)$ \\
First additional test request & $191(173-210)$ \\
Second additional test request & $10(8-14)$ \\
Third additional test request & $0(0-1)$ \\
Additional sample & $271(233-295)$ \\
\hline
\end{tabular}

Additional tasks are expressed in absolute numbers and per 1000 tests requested.

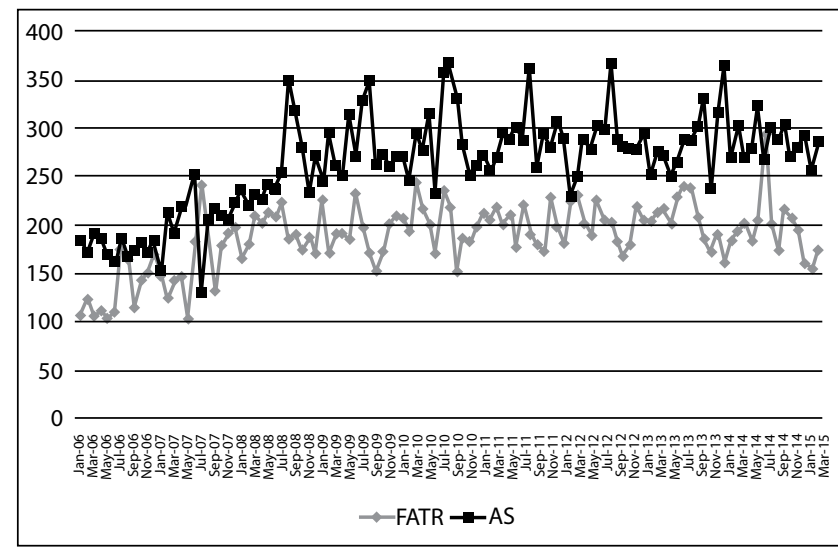

Figure 1. Temporal evolution of different additional tasks performed by Stat laboratory technicians.

FATR - first additional test request, AS - additional sample. The number of FATR and AS on a monthly basis is shown along the study period.

In spite of the seasonal variations in the workload and additional technician tasks, the TAT did not show this variation between summer and the rest of the year when expressed as median or $90^{\text {th }}$ percentile (P90), complying always with our 30 minutes indicator target.

\section{Discussion}

This is the first study that counts and calculates additional tasks conducted by SL technicians and compares TAT. The technicians in the SL accomplished a significant number of additional tasks that effectively increased the SL workload. Addi-

TABLE 2. Comparison of workload, additional tasks and TAT between summer and the rest of the seasons.

\begin{tabular}{lccc}
\hline & Summer & Rest of the seasons & $\mathbf{P}^{*}$ \\
\hline Total test (workload) & 45,496 & 44,555 & 0.019 \\
Total additional tasks / 1000 tests performed & $(43,925-47,922)$ & $(42,134-46,608)$ & $<0.001$ \\
TAT P90, minutes & $11.4(10.4-11.9)$ & $10.0(9.3-10.9)$ & $52(50-55)$ \\
TAT P50, minutes & $51.4(50-53)$ & $29.2(27-31)$ & 0.163 \\
\hline
\end{tabular}

Additional tasks are expressed in absolute numbers and per 1000 tests requested as median and interquartile range. P90 - $90^{\text {th }}$ percentile, $\mathrm{P} 50-50^{\text {th }}$ percentile.

*Significant differences tested with the Mann-Whitney $U$ test. $P<0.05$ was considered statistically different. 
tionally, laboratory requests kept increasing over the period of 10 years. However, even with the observed seasonal variation in the number of additional tasks, that did not impact the TAT results, that were maintained over the years. The number of "additional tasks" during 10 years was stable in spite of the higher workload, probably because of the annual session to educate physicians for a better use of SL.

It is true that the clinical laboratory and the ED must work together to take care of patients quickly and effectively (1), using all the tools at our disposal to accomplish the ED physicians expectations such as TAT (4). However, it is also certain that process control is needed to reduce the laboratory turnaround time (6). It is therefore necessary to monitor and control every task that may affect response time, especially in the ED, where acute patients need prompt and accurate response.

To this regard, as the urgent test definition states a test whose results may imply an immediate therapeutic action (9) - the potential damage of a laboratory error in this setting can be immediate. Interestingly enough, increasing volume of ED patients results in longer laboratory processing times. Prolonged laboratory TAT leads, in turn, to delayed diagnosis of potentially severe and life threatening conditions in the acutely ill patients, which has a negative impact in clinician decision-making and initiation of timely treatment (10). As a result, one must be very cautious when deciding on the resources in the SL, to improve laboratory and pa-

\section{References}

1. Lewandrowski K. How the clinical laboratory and the emergency department can work together to move patients through quickly. Clin Leadersh Manag Rev 2004;18:155-59.

2. Department of Health. The NHS Plan: a plan for investment, a plan for reform. Available at: http://webarchive.nationalarchives.gov.uk/+/www.dh.gov.uk/en/publicationsandstatistics/publications/publicationspolicyandguidancel dh_4002960. Accessed April 15th 2015.

3. Salinas $M$, López-Garrigós $M$, Santo-Quiles A, Gutierrez M, Lugo J, Lillo R, et al. Customising turnaround time indicators to requesting clinician: a 10-year study through balanced scorecard indicators. J Clin Pathol 2014;67:797-801. http:// dx.doi.org/10.1136/jclinpath-2014-202333 tient safety. In that scenario, one also needs to be aware of the additional variables that may affect the SL workflow, before TAT is slowed down.

The study has several limitations. First, there are some other additional tasks performed by the laboratory technician in SL that have not been collected, such as phone calls. Second, the use of troponin as indicator of TAT and efficiency of the SL, could not apply to laboratories where troponin is released quickly through a point of care testing measurement. Lastly, the study results could be difficult to extrapolate to those laboratories where the $\mathrm{SL}$ is integrated in central laboratory, or those with technological advances such as Computerized Provider Order Entry.

In conclusion, the technicians in the SL accomplished a significant number of additional tasks regarding the request of additional tests or the submission of additional samples that did not affect the TAT results. Considering that results of stat tests may imply an immediate therapeutic action, it is important to acknowledge the real SL technicians workload, including additional tasks that increase the effective SL workload. Furthermore, by controlling additional tasks that may affect the service provided by the laboratory, we are able to still maintain/improve this service, and hence the contribution to the ED patients and their safety.

\section{Potential conflict of interest}

None declared.

4. Salinas M, López-Garrigós M, Gutiérrez M, Lugo J, Llorca F, Uris J. Stat laboratory timeliness management according to clinician needs. Clin Chem Lab Med 2011;49:331-3. http:// dx.doi.org/10.1515/CCLM.2011.042

5. The royal college of Pathologist. Key performance indicators - Proposals for implementation. Available at: https://www. rcpath.org/resourceLibrary/key-performance-indicators--proposals-for-implementation-.html. Accessed April 15th 2015.

6. Carraro P, Plebani M. Process control reduces the laboratory turnaround time. Clin Chem Lab Med 2002;40:421-2. http:// dx.doi.org/10.1515/CCLM.2002.068 
7. Fei $Y$, Zeng R, Wang W, He F, Zhong K, Wang Z. National survey on intra-laboratory turnaround time for some most common routine and stat laboratory analyses in 479 laboratories in China. Biochem Med (Zagreb) 2015;25:213-21. http://dx.doi.org/10.11613/BM.2015.021

8. Hawkins RC. Laboratory turnaround time. Clin Biochem Rev 2007;28:179-94.
9. Volmar KE, Wilkinson DS, Wagar EA, Lehman CM. Utilization of stat sest priority in the clinical laboratory: A College of American Pathologists q-Probes Study of 52 institutions. Arch Pathol Lab Med 2013;137:220-7. http://dx.doi. org/10.5858/arpa.2012-0100-CP

10. Hwang U, Baumlin K, Berman J, Chawla NK, Handel DA, Heard $K$, et al. Emergency department patient volume and troponin laboratory turnaround time. Acad Emerg Med 2010;17:501-7. http://dx.doi.org/10.1111/j.15532712.2010.00738.x 